\title{
GROUP A STREPTOCOCCUS DIAGNOSTIC METHODS AND VIRAL CO-INFECTIONS IN PHARYNGITIS
}

Lauri Ivaska, ${ }^{a}$ Jussi Niemelä, ${ }^{a}$ Matti Waris, ${ }^{b}$ Tytti Vuorinen, ${ }^{b}$ Niina Putkuri, ${ }^{c}$ Kaisu Rantakokko-Jalava, ${ }^{c}$ Ville Peltola ${ }^{a}$

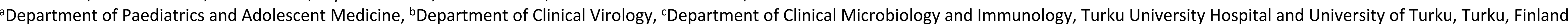

\section{Background}

Co-detection of group A streptococcus (GAS) and viruses in acute pharyngitis has not been well documented. We aimed to study the occurrence of viral co-infection in relation to GAS diagnostic method.

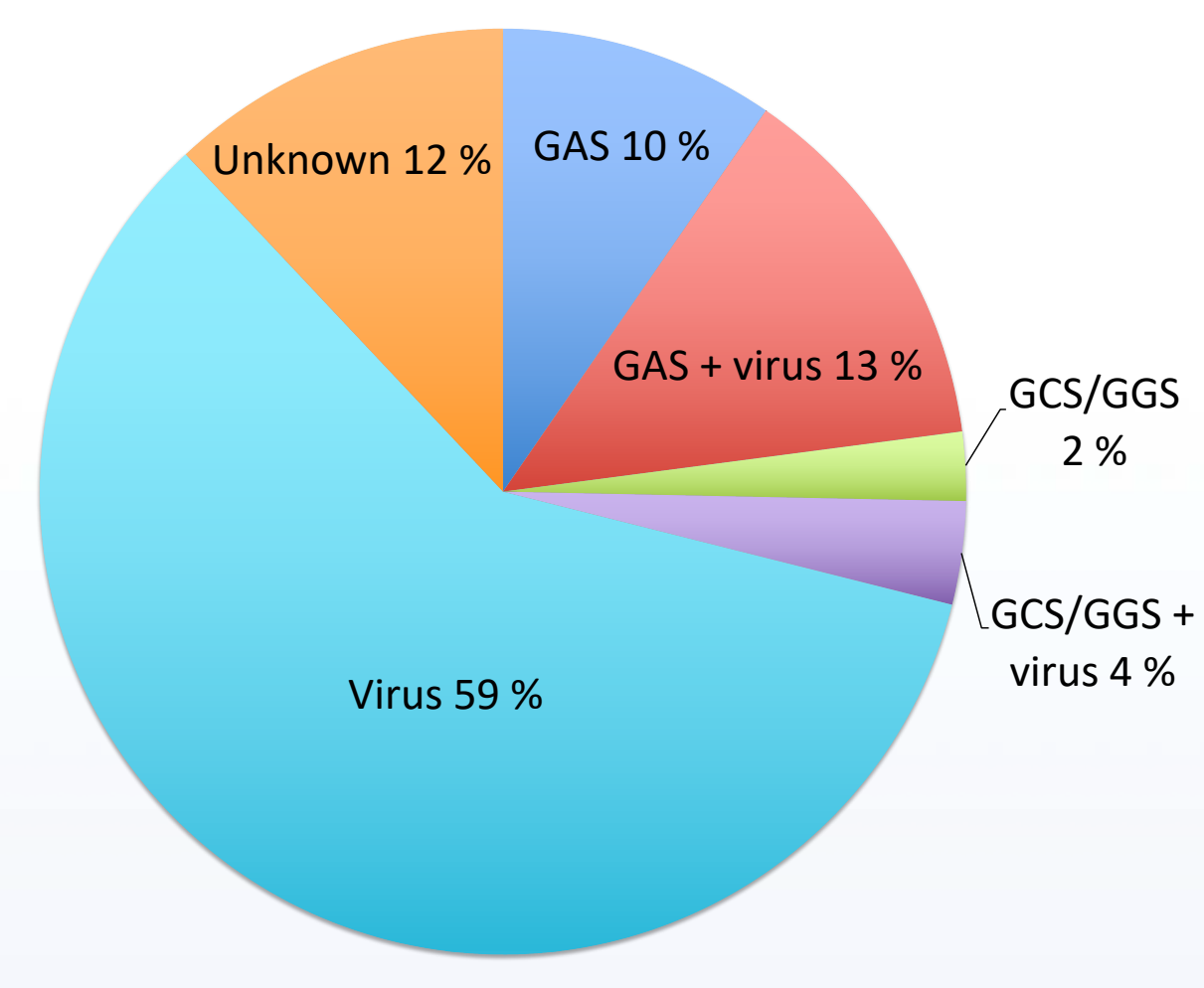

Figure 1. Microbiological aetiology of pharyngitis in 83 children. GAS, group A streptococcus; GCS, group C streptococcus; GGS, group G streptococcus (Ivaska L et al. J Infection 2017;74:385-392.)

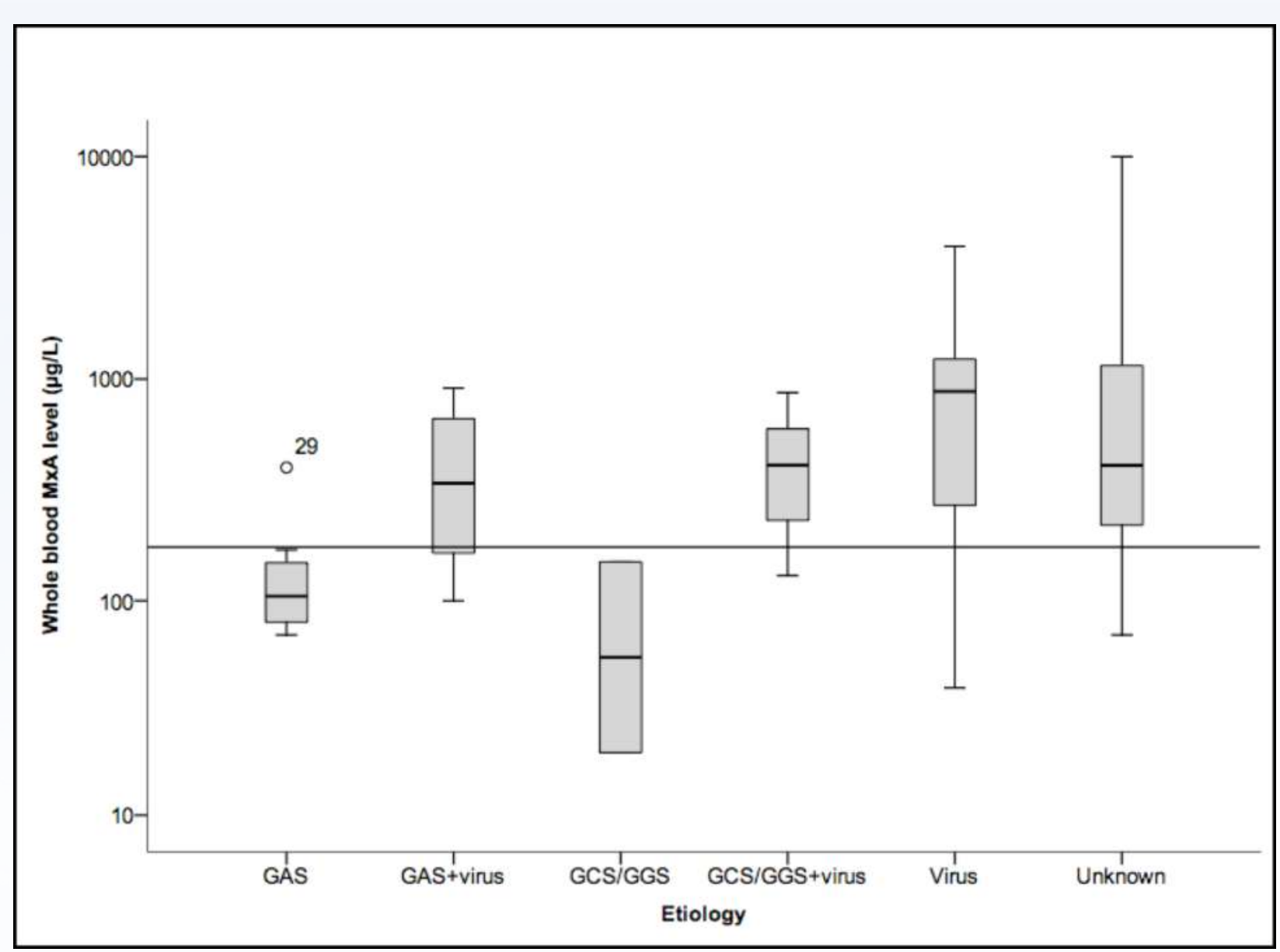

Figure 2. Blood myxovirus resistance protein $A(M \times A)$ levels in the different aetiological groups of 83 children with pharyngitis. GAS, group A streptococcus; GCS, group C streptococcus; GGS, group G streptococcus (Ivaska L et al. J Infection 2017;74:385-392.)

\section{Methods}

This was part of a prospective study done in an emergency department (ED) in febrile children 1-16 years of age with pharyngitis. Throat swabs and blood samples were collected and testing for GAS was performed by 2 different rapid antigen detection tests (RADTs; StrepTop and mariPOC) in the ED and by throat culture in laboratory. In addition, frozen throat swabs were tested for GAS by 2 different nucleic acid amplification tests (NAAT; Focus $3 \mathrm{M}$ and Illumigene). Virus diagnostics was performed by NAAT and serology, and interferon response to virus infection was estimated by myxovirus resistance protein $A(M x A)$ blood concentration.

\section{Results}

In total, 83 children (median age 5.5 years; interquartile range 3.2-12.2) were recruited in the study. Specimens from 78 and 48 patients were available for RADT and NAAT, respectively (Figure 3). In comparison with throat culture results, sensitivities and specificities of GAS diagnostic tests were $72 \%$ and $100 \%$ for StrepTop, $100 \%$ and $72 \%$ for mariPOC, $91 \%$ and $87 \%$ for Focus $3 \mathrm{M}$, and $91 \%$ and $96 \%$ for Illumigene, respectively (Table 1 ). In GAS positive patients, virus detection and elevated blood MxA level ( $\geq 175 \mu \mathrm{g} / \mathrm{L}$ ) were more frequent when GAS diagnosis was based on mariPOC (54\%) than when it was based on throat culture (44\%) results (Figure 4).

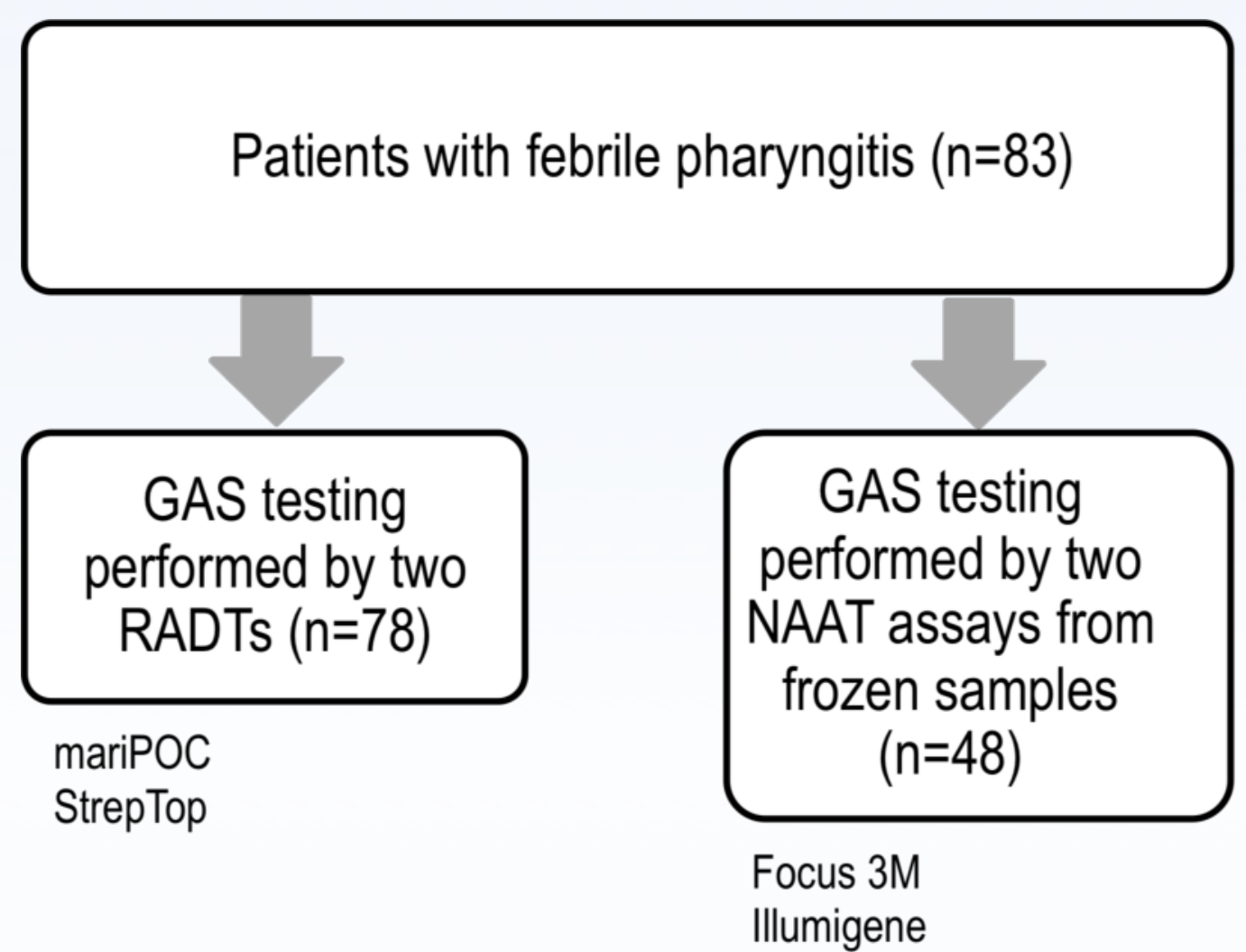

Figure 3. Study flow chart. GAS, group A streptococcus; RADT, rapid antigen detection test; NAAT, nucleic acid amplification technique

\section{Conclusions}

In comparison with throat culture, diagnostic performance of different GAS tests varies greatly. In children with febrile pharyngitis, enhanced sensitivity of the GAS assays seems to be associated with increased codetection of viruses and interferon responses questioning the significance of GAS detection in these patients.

Table 1. Number of group A streptococcus (GAS) positive samples and diagnostic performance of four different GAS assays versus culture on blood agar plate from throat swabs; RADT, rapid antigen detection test NAAT nucleic acid amplification technique; PPV, positive predictive value; NPV, negative predictive value

\begin{tabular}{|c|c|c|c|c|c|}
\hline Diagnostic method & GAS positive samples, $n(\%)$ & Sensitivity & Specificity & PPV & NPV \\
\hline Throat culture $(n=83)$ & $19(23)$ & & & & \\
\hline \multicolumn{6}{|l|}{ RADT $(n=78)$} \\
\hline StrepTop ${ }^{\circledR}$ & $13(17)$ & 72 & 100 & 100 & 92 \\
\hline mariPOC & $35(45)$ & 100 & 72 & 51 & 100 \\
\hline \multicolumn{6}{|l|}{ NAAT $(n=48)$} \\
\hline Focus $3 \mathrm{M}^{\bullet}$ & $15(31)$ & 91 & 87 & 67 & 97 \\
\hline |llumigene ${ }^{\circledR}$ & $11(23)$ & 91 & 97 & 91 & 97 \\
\hline
\end{tabular}

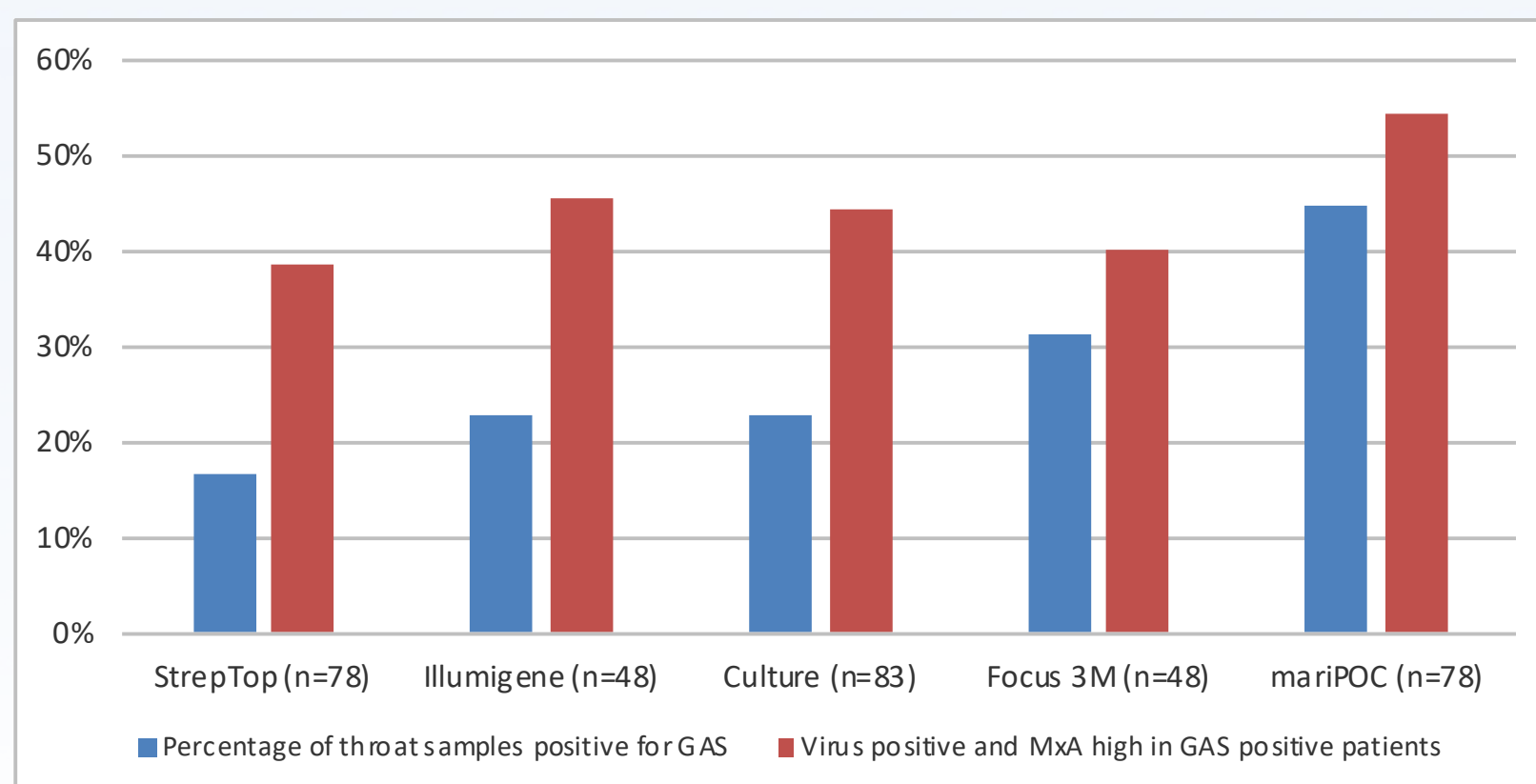

Figure 4. Proportions of group A streptococcus (GAS) positive throat samples (blue bars) and children with GAS-positive pharyngitis with virus detection and elevated blood myxovirus resistance protein $A$ (MxA) levels ( $\geq 175 \mu \mathrm{g} / \mathrm{L}$ ) (red bars) with different diagnostic methods. 